Mens

revue d'histoire intellectuelle de l'Amérique française

\title{
Une lettre de Louis Lachance, o.p. à Lionel Groulx (ca 1941)
}

\section{Yves Bégin}

Volume 1, numéro 2, printemps 2001

URI : https://id.erudit.org/iderudit/1024448ar

DOI : https://doi.org/10.7202/1024448ar

Aller au sommaire du numéro

Éditeur(s)

Centre de recherche en civilisation canadienne-française

ISSN

1492-8647 (imprimé)

1927-9299 (numérique)

Découvrir la revue

Citer ce document

Bégin, Y. (2001). Une lettre de Louis Lachance, o.p. à Lionel Groulx (ca 1941).

Mens, 1(2), 163-169. https://doi.org/10.7202/1024448ar d'utilisation que vous pouvez consulter en ligne.

https://apropos.erudit.org/fr/usagers/politique-dutilisation/ 
INÉDIT $^{1}$

Une lettre de Louis Lachance, o.p. ${ }^{2}$ à Lionel Groulx ${ }^{3}$ [s.d.] ${ }^{4}$

Édition critique établie par

Yves Bégin

Département d'histoire

Université de Montréal

\section{PRÉSENTATION}

Cette lettre du père Louis Lachance provient du Fonds Lionel-Groulx des Archives du Centre de recherche Lionel-Grouls. Lachance répond vraisemblablement à une question que lui aurait posée l'abbé Groulx ${ }^{6}$. Ce dernier lui aurait demandé des «distinctions philosophiques» sur les devoirs de patriotisme que doivent observer les Canadiens français à l'égard de la nation canadiennefrançaise, d'une part, et de l'État fédéral, d'autre part.

On ne connait pas la nature exacte de la relation entre les deux hommes. On sait que Groulx a conservé dix lettres du père Lachance (entre 1934 et 1961), mais on ne retrouve pas le nom de ce dernier dans l'index des Mémoires de Groulx, pourtant assez exhaustif. En fait, il semble que Groulx a fait appel à Lachance à titre de "conseiller théologique» à quelques reprises. Ainsi, à au moins deux occasions dans cette correspondance, Lachance se prononce sur le caractère orthodoxe de textes que Groulx lui aurait fait lire ${ }^{7}$. Au cours des années vingt et trente, Groulx avait souvent fait appel à son ami le théologien Louis-Adolphe Pâquet pour ce gente de service. Mais Pâquet, né en 1859, commence à se faire vieux au milieu des années trente et Groulx est probablement à la recherche d'un «remplaçant». Peut-être voit-il en Louis Lachance un candidat potentiel. Il ne faut donc pas s'étonner outre mesure de voir Groulx demander l'avis d'un théologien sur l'orthodoxie de sa pensée. Ce qui nous apparait plus étonnant en revanche c'est que Groulx, considéré comme le chef de file et principal animateur du mouvement nationaliste depuis le milieu des années vingt, demande ici à un jeune philosophe de vingt et un ans son cadet une opinion philosophique (pratiquement une leçon en l'occurrence) au sujet du nationalisme. Lachance s'étonne d'ailleurs de voir celui qu'il considère son umaître»" questionner l'élève sur ces matières. 
Si Groulx connaissait Lachance au moins depuis 1934 (date de la première lettre de Lachance à Groulx), il l'aura probablement davantage remarqué lors de la publication de son essai Nationalisme et religion. On sait que Groulx a lu cet ouvrage et il semble l'avoir appré$c e^{8}$. En droite ligne avec la volonté de urelégitimation» du nationalisme du clergé canadien-français à la suite de la condamnation du nationalisme outrancier par le pape Pie XI dans l'affaire de L'Action francaise de Paris en 1926, l'objectif de l'auteur était d'analyser, à la lumière de la pensée de saint Thomas d'Aquin, les rapports entre le nationalisme et la religion catholique et de prouver leur compatibilité, voire la nécessaire dépendance de l'un par rapport à l'autre. Pour Lachance en effet, la raison, la foi et la morale catholique doivent encadrer le nationalisme pour empêcher qu'il ne dévie ou qu'il soit utilisé pour la promotion d'une doctrine matérialiste, raciste et violente. Son essai vise à offrir aux nationalistes canadiens-français une pensée nationaliste rationnelle d'une parfaite orthodoxie sur le plan catholique. Groulx, souvent accusé de faire la promotion d'un nationalisme passionné, et même outrancier, émettait depuis longtemps le désir de formuler une doctrine nationaliste irréprochable, propre à fortifier la volonté des siens et à convaincre ses adversaires de la légitimité de son action. Il ne pouvait qu'approuver le travail du père Lachance.

Le contenu de cette lettre reflète les préoccupations de Groulx concernant la légitimité de son nationalisme, et aussi une volonté d'articuler son nationalisme, bien canadien-français, à la réalité politique canadienne. Deux questions, en particulier, semblent avoir poussé l'abbé Groulx à faire appel à Lachance : le Canada est-il une patrie commandant des devoirs de piété (patriotisme) et est-il légitime de se consacrer d'abord au bien de la province et ensuite seulement à celui du Canada? 


\section{Séminaire des Saints-Apôtres \\ Sherbrooke, P.Q.}

Monsieur l'abbé Lionel Groulx, Outremont

\section{Cher M. Groulx,}

Si je n'avais l'habitude de consulter mes élèves, je comprendrais mal que vous vous adressiez à moi à propos de questions où vous avez été mon maitre. Mais je comprends que vous voulez des distinctions philosophiques et je m'exécute sans plus de façons.

$\grave{A}$ mon sens, le Canada n'est pas tant une patrie qu'une entité politique ou juridique. On est citoyen canadien. En conséquence, les devoirs que nous avons envers le tout sont des devoirs de justice et non pas de patriotisme proprement dits.

Nos devoirs de piété doivent aller, comme vous le dites, à ceux qui sont à la source de notre être naturel et culturel : in quantum sunt principia in esse'. Le motif, c'est que l'effet conscient éprouve un lien d'obligation envers sa cause, et dans la mesure où celle-ci fut excellente, dans la mesure où ce qu'elle a légué est précieux ${ }^{10}$.

La question du territoire jouait énormément chez les Anciens, étant donné qu'ils se croyaient autochtones, issus de la terre. ( $\mathrm{La}$ motte de terre qu'ils emportaient en exil). Toutefois dans la notion chrétienne de piété, c'est surtout la personnalité morale de la nationalité, ses grandeurs et ses dévouements qui commandent la piété. Le territoire n'entre en ligne de considération que comme partie du patrimoine de la nation".

Par conséquent, dans l'ordre du sentiment et de la piété, c'est la nation qui prime. Ses biens de culture qui ne sont pas incompatibles avec la vie publique de l'État sont à conserver. Ce qui nous crée des devoirs stricts. Pour moi, le sentiment canadien, - chose encore assez vague, - ne doit jamais l'emporter sur le sentiment canadien français ${ }^{12}$.

La comparaison entre nos devoirs envers la Province et l'État doit s'établir sur le plan de la justice sociale, c'est-à-dire de ${ }^{13}$ celle qui a pour objet le bien commun. Et s'il est possible de philosopher sur cette entité bâtarde qui est notre constitution, voici comment il faudrait le faire, je pense bien. 
1- Il est parfaitement juste de dire que la cause de la confédération fut et demeure l'insuffisance des parties contractantes.

2- Le bien commun résulte forcément de la coordination du bien des parties. Il s'identifie matériellement avec celui-ci. Formellement, il s'en distingue, puisqu'il enchâsse dans un ordre le bien de chacune des parties. L'ordre organise, embrasse tous les biens particuliers.

3- Bien que, historiquement, les pouvoirs du fédéral lui aient été octroyés par les provinces et bien que cette distribution ait été étrange et n'ait pas constitué vraiment une hiérarchie ${ }^{14}$ (une entente? un compromis?), il faut cependant admettre, je crois, que l'État central, une fois constitué, détient de sa nature même ou des fonctions qui découlent de son essence, des pouvoirs qui lui sont propres, encore que ces pouvoirs ne soient que relatifs à la coordination des biens particuliers, en quoi consiste le bien commun ${ }^{15}$.

4- Il est impossible que le bien commun bien entendu (ne) s'oppose à celui des parties bien entendu. Le bien de l'ensemble retentit sur celui des parties et en permet la pleine réalisation.

5- Je touche ici à votre question : relations entre le bien du tout et le bien des parties. Pour S. Thomas ${ }^{16}$, la partie n'a jamais pour fin propre ou intrinsèque le bien du tout. $\mathrm{Sa}$ fin immédiate et à elle est son bien propre. Celui-ci est condition indispensable du bien commun. Par conséquent il faut dire que la partie doit rechercher immédiatement ${ }^{17}$ son bien propre et médiatement le bien commun; bien plus, c'est en recherchant son bien propre qu'elle concourt au bien commun. Conséquence, en travaillant au bien de la province, c'est le meilleur moyen de contribuer à la réalisation du bien commun ${ }^{18}$.

Inversement le fédéral, lui, doit travailler immédiatement à son oeuvre d'unité et de coordination et médiatement au bien des provinces. Pour éviter les conflits, s'en tenir à la lettre de la constitution ${ }^{19}$.

6- L'individu, en particulier, peut-il se consacrer immédiatement au bien de la partie ou de la province. Certainement, s'il juge ${ }^{20}$ il le doit même, s'il juge que le bien de la partie est condition indispensable de la conservation de nombre d'autres biens, qui ne sont pas à proprement parler politiques, mais moraux, culturels et religieux. 
Je tire quelques conclusions.

1- Envers le Fédéral, il n'est pas question de patriotisme, si ce n'est dans la mesure où tout le Canada est notre patrimoine historique. On ne donne pas son coeur à une entité juridique.

2- Nous avons des devoirs de justice sociale envers la province, dans la mesure où son bien propre l'exige.

3-Nous avons des devoirs de justice sociale envers le Canada (taxes, service militaire, etc.), dans la mesure où ${ }^{21}$ le bien du tout, et par ricochet celui des parties, est concernéé ${ }^{22}$.

Même dans l'accomplissement de ses devoirs envers le $\mathrm{Ca}$ nada, il n'y a rien de pervers à motiver ses actes par le bien de la Province qui se trouve médiatement en jeu. Il est toujours légitime, et méme normal, de vouloir le bien du tout à travers celui de la partie ${ }^{23}$.

J'ai bien honte de vous adresser ces élucubrations, mais je m'empresse de le faire, afin de vous donner le temps de discuter certains points si vous le désirez ${ }^{24}$.

Ensuite, vous savez mieux que moi qu'aucune théorie ne colle parfaitement sur notre appareil gouvernemental. On ne peut pas vraiment dire que du Provincial au Fédéral, il y a hiérarchie. Sur bien des points, le Provincial est au-dessus ${ }^{25}$ du fédéral. La hiérarchie, dans notre cas, n'est souvent que spatiale ${ }^{26}$.

Encore une fois, je vous prie de m'excuser et d'agréer l'expression de mes sentiments très cordiaux.

En la S. Vierge

Louis Lachance, o.p.

\section{NOTES}

${ }^{1}$ Nous aimerions remercier le Centre de recherche Lionel-Groulx ainsi que Jean Fournier de l'Administration provinciale des Pères dominicains pour leur concours.

${ }^{2}$ Louis Lachance, o.p. (1899-1963). Père dominicain, professeur de philosophie et intellectuel nationaliste, né à Saint-Joachim de Montmorency. Professeur de philosophie à l'Université d'Ottawa (1931-1936) et à l'Angelicum à Rome (1936-1938), il participe au début des années trente à la fondation de l'Institut d'études médiévales d'Ottawa ainsi qu'à celle du Grand séminaire des Saints-Apôtres de Sherbrooke. Maître en sacrée théologie en 1944, il est membre fondateur la même année de l'Académie canadienne-française. Professeur de philosophie morale et sociale à l'Université 
de Montréal depuis 1944, il devient doyen de la même faculté en 1960. Philosophe reconnu internationalement, il est l'auteur de plusieurs ouvrages de philosophie, dont Nationalisme et religion (1936) et $L$ Droit et les droits de l'homme (1959).

${ }^{3}$ Lionel Groulx (1878-1967). Prêtre, historien, romancier et intellectuel nationaliste, il fut le chef de file du mouvement nationaliste canadien-français du milieu des années vingt au début des années cinquante. Professeur d'histoire à l'Université de Montréal de 1915 à 1949, il fut concurrement un auteur prolifique, publiant au cours de sa vie de nombreux ouvrages d'histoire, dont son Histoire du Canada frangais depuis la découverte, deux romans, ainsi que quelques dizaines d'articles et de textes de conférences. Il a aussi dirigé L'Action frangaise de Montréal entre1920 et 1927 et fondé la Revue d'bistoire de l'Amérique frangaise en 1947.

'La lettre n'est pas datée. Pourtant, certains indices nous laissent croire qu'elle daterait de 1941. Tout d'abord, Lachance a été professeur au Grand séminaire des Saints-Apôtres à Sherbrooke et l'en-tête de la lettre est celle du Séminaire, fondé en 1939. Elle ne peut donc avoir été écrite avant 1939. De plus, elle ne peut vraisemblablement pas dater d'après 1943 puisque à cette date Groulx devient chanoine, et Lachance écrira toujours dans les lettres datées d'après 1943 «M. le chanoine» et non «M. l'abbé» comme dans la lettre. Enfin, le 16 septembre 1941, Groulx écrit dans une lettre à Lachance : «Je voulais toujours accuser réception des bonnes notes que vous m'avez fournies le printemps derniens. Il nous apparait raisonnable de penser que Groulx fait référence à cette lettre de Lachance. Plus loin dans la lettre, il écrit aussi : «Je crois toujours que vous devriez rédiger, là-dessus, une consultation philosophique ou politique. Vous ne sauriez croire comme la confusion des idées trouble les meilleurs esprits, surtout parmi la jeunesse. Les politiciens jouent de leur mieux, dans ce chaos, avec le résultat que vous savez.» Cette suggestion correspond au contenu de la lettre de Lachance que nous présentons. Lettre de Lionel Groulx à Louis Lachance, Outremont, 1941, 1 p dact. Archives du Centre de Recherche Lionel-Groulx, Fonds Lionel-Groulx, P1/A, 1939.

${ }^{5}$ Lettre de Louis Lachance à Lionel Groulx, [s.d., probablement 1941], 6 p. mss., ACRLG, FLG, P1/A, 1939. Les soulignés sont du père Lachance.

${ }^{6}$ Nous n'avons pu retracer cette lettre ni dans le Fonds Lionel-Groulx ni dans celui du Père Lachance.

${ }^{7}$ "J'ai lu vos excellentes pages et je ne puis que vous en féliciter. Elles sont non seulement "orthodoxes", mais constitue [si] une directive ferme et très opportune.», Lettre de Louis Lachance à Lionel Groulx, Sherbrooke, [s.d., probablement 1941], 1 f. mss., ACRLG, FLG, P1/A, 1939 (nous datons cette lettre en fonction du fait que Lachance y répond à une lettre de Groulx datée de septembre 1941); «J'ai été vivement intéressé par votre étude de $\mathrm{M}^{\mathrm{gr}}$ Plessis. Du point de vue théologique, tout me semble parfait..), lettre de Louis Lachance à Lionel Groulx, Sherbrooke, [s.d., probablement 1943 ou 1944], 1 f. (recto-verso), ACRLG, FLG, P1/A, 1939 (nous datons cette lettre en fonction du fait que Lachance écrit "Cher M. le Chanoine» - Groulx a reçu le titre de chanoine honoraire en 1943 - et parce que le papier utilisé porte l'en-tête du Séminaire des Saints-Apôtres de Sherbrooke, qu'il quitte en 1944 pour l'Université de Montréal. Il est peu probable qu'il ait utilisé ce papier officiel après son départ).

${ }^{8}$ En effet, lors d'une causerie faite en septembre 1936, il déclare: «Énonçons un principe doctrinal que j'emprunte au livre lumineux du Père Louis Lachance, o.p., Nationalisme et religion [...]", voir L. Groulx, «Labeurs de demain» (1936), dans Directives, Montréal, Éditions du Zodiaque, 1937, p. 128. 
' $\mathrm{A}$ proportion qu'ils sont à la source de l'être». Il s'agit d'une répétition de la phrase qui précède, l'idée de proportionnalité en plus. Nous tenons à remercier les professeurs Pierre Trépanier et Pierre Boglioni pour leurs suggestions.

${ }^{10} \mathrm{Groulx}$ a noté en marge : «le milieu national est générateun.

"Groulx a marqué le passage d'un trait dans la marge.

"Écrit «canadien français» (sans trait d'union. Groulx a noté en marge : «n.b.».

${ }^{13}$ Lachance avait écrit «sue, l'a biffé pour écrire «de» par-dessus.

${ }^{14}$ Écrit «hierarchie» (sans accent).

${ }^{15}$ Groulx a marqué la dernière phrase d'un «X » dans la marge.

${ }^{16}$ Saint-Thomas d'Aquin (1228-1274). Théologien et philosophe italien, il enseigna à l'Université de Paris et à Naples. Canonisé en 1323 et proclamé docteur de l'Église en 1923, il est, entre autres, l'auteur de la Somme théologique. Il est l'un dés plus grands représentants de l'ordre des Dominicains. Une partie de son enseignement fut condamnée en 1277, mais aux environs de 1300, le thomisme (qui se fonde sur l'articulation entre la foi et la raison) s'est rétabli de cette condamnation et son influence continue de croître jusque vers le milieu du XVe et au XVII' siècles, mais le cartésianisme le concurrence et il tombe en décadence. Une troisième période importante commence au milieu du XIX' siècle et son renouveau est consacré par le pape Léon XIII en 1879. Le néo-thomisme connaît ensuite ses plus beaux jours avec Étienne Gilson, Jacques Maritain et Garrigou-Lagrange.

${ }^{17 E ́ c r i t ~ u i m m e d i a t e m e n t » ~(s a n s ~ a c c e n t) . ~}$

${ }^{18} \mathrm{Groulx}$ a noté dans la marge : «La province doit chercher immédiatement son bien, médiatement le bien commun».

${ }^{19} \mathrm{Groulx}$ a noté dans la marge : «L'État fédéral immédiatement la coordination, médiatement le bien des provinces».

${ }^{20}$ Biffé par Lachance.

${ }^{21}$ Écrit «ou» (sans accent).

${ }^{22}$ Lachance avait d'abord écrit «menacé» et l'a biffé d'un trait.

${ }^{23}$ Passage souligné par Groulx.

${ }^{24} \mathrm{Cette}$ phrase suggère que Groulx avait besoin de ces précisions en vue d'un événement rapproché.

${ }^{25}$ Écrit : «au dessus» (sans trait d'union).

${ }^{26}$ Écrit : «spaciale». 